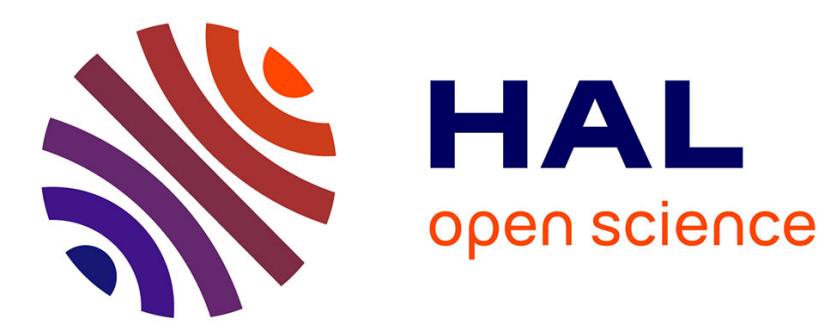

\title{
Model-based interpretation of cardiac beats by evolutionary algorithms: signal and model interaction.
}

Alfredo Hernández, Guy Carrault, Fernando Mora, Alain Bardou

\section{To cite this version:}

Alfredo Hernández, Guy Carrault, Fernando Mora, Alain Bardou. Model-based interpretation of cardiac beats by evolutionary algorithms: signal and model interaction.. Artificial Intelligence in Medicine, 2002, 26 (3), pp.211-35. 10.1016/S0933-3657(02)00078-7 . inserm-00134397

\section{HAL Id: inserm-00134397 https://www.hal.inserm.fr/inserm-00134397}

Submitted on 27 Mar 2007

HAL is a multi-disciplinary open access archive for the deposit and dissemination of scientific research documents, whether they are published or not. The documents may come from teaching and research institutions in France or abroad, or from public or private research centers.
L'archive ouverte pluridisciplinaire HAL, est destinée au dépôt et à la diffusion de documents scientifiques de niveau recherche, publiés ou non, émanant des établissements d'enseignement et de recherche français ou étrangers, des laboratoires publics ou privés. 


\author{
Alfredo I. Hernández*, Guy Carrault*, Fernando Mora**, Alain Bardou* \\ *Laboratoire Traitement du Signal et de l'Image. Université de Rennes 1.
} Campus de Beaulieu Bât 22. 35042 Rennes, France.

**Grupo de Bioingeniería y Biofísica Aplicada. Universidad Simón Bolívar.

Apartado 89000. Caracas, Venezuela.

Corresponding author: Alfredo I. Hernández.

E-mail: Alfredo.Hernandez@univ-rennes1.fr

\title{
ABSTRACT
}

This paper presents a new approach for cardiac beat interpretation, based on a direct integration between a model and observed ECG signals. Physiological knowledge is represented by means of a semi-quantitative model of the cardiac electrical activity. The interpretation of cardiac beats is formalized as an optimization problem, by minimizing an error function defined between the model's output and the observations. Evolutionary algorithms are used as the search technique in order to obtain the set of model parameters reproducing at best the observed phenomena. Examples of model adaptation to three different kinds of cardiac beats are presented. Preliminary results show the potentiality of this approach to reproduce and explain complex pathological disorders and to better localize their origin.

Keywords: Cardiac rhythm interpretation, Model-based systems, Evolutionary Algorithms.

\section{INTRODUCTION}

Computer-assisted cardiac rhythm interpretation has been considered a very important issue in the biomedical field. Although there have been major contributions to this area, the poor performance of 
current automatic systems is an important drawback in their introduction into routine clinical practice. During the last decade, large efforts have been directed to integrate medical knowledge into the interpretation process, in order to develop “intelligent monitoring" systems. Despite its limitations, this approach has shown promise in improving automatic interpretation of clinical data [23].

Integration of medical knowledge can be done by distinguishing two main representation levels: "shallow" or experiential knowledge and "deep" knowledge. By directly mapping experiential knowledge into a computer-assisted system, an expert or knowledge-based system (KBS) can be implemented. Most of the current systems for medical diagnosis have been developed following this methodology. Deep knowledge can be introduced by means of model-based systems (MBS), in which a model of the underlying physiology is built to obtain a useful explanation of the observation, leading to a comprehensive and compact knowledge representation that can be applied for diagnosis or for prediction. A stepping-stone in the practical application of MBS to dynamic systems has been the implementation of the interface between model and observations, as well as the stages of hypothesis generation and machine reasoning, often difficult to define. This paper presents a new approach for the interpretation of cardiac beats, combining model-based reasoning and evolutionary computing, which

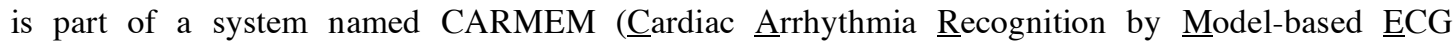
Matching), being developed in our laboratory [9]. This approach directly integrates observed ECG signals with a semi-qualitative cardiac model by: $i$ ) defining an error function between the model's output and the observations and ii) applying evolutionary algorithms to adapt the model parameters in order to minimize the observed error.

After a brief review of current MBS for cardiac arrhythmia interpretation, the proposed approach is described. Three beat interpretation examples are also presented, showing the potentialities and current limitations of the approach.

\subsection{STATE OF THE ART}

The initial efforts towards the development of a model-based system for the analysis and diagnosis of cardiac arrhythmias can be summarized into four representative systems, each one possessing its own peculiarities: "KARDIO" [2], the set of "Ticker" models [12], "EINTHOVEN" [25] and "HOLMES" [6]. 
KARDIO is a well-known system, often used as a textbook example of machine-generated knowledge. It consists of a set of rules defining deep electrophysiological relations that permits the generation of new knowledge about the observed phenomena. The other three systems are based on spatio-temporal electrophysiological models of the cardiac activity, with varying degrees of detail. They also present a reasoning mechanism, generally based on a hypothesize-and-test paradigm, allowing the generation of a set of explanations, followed by a pruning phase, in order to keep the most significant interpretations. However, among the systems mentioned, only EINTHOVEN presents a complete reasoning structure that has been evaluated on a number of different rhythms [26].

Besides the limitation in the explanation of pathological rhythms, current MBS's lack a practical way of associating the modeled knowledge with the clinical observations. Usually, a set of hand-made annotations about occurrence of atrial and ventricular activities are employed, accompanied, in some cases (EINTHOVEN and HOLMES), with a qualitative morphological description of each observed ECG wave. The absence of a direct automatic integration of model and observed phenomena represents a huge limitation of current MBS in their on-line interpretation of cardiac activity.

\subsection{PROPOSED APPROACH}

Two major abstraction levels can be identified in any intelligent monitoring system (see Figure 1): A lower abstraction level, dedicated to the preprocessing of the observed activity and a higher abstraction level, concerned with the interpretation of the observed phenomena.

\section{INSERT Figure 1 HERE.}

In the proposed system for cardiac diagnosis, the lower level is responsible for detecting the main cardiac events, for example, occurrence of atrial and ventricular activities ( $P$-wave and $Q R S$ complex of the ECG, respectively). Low level signal processing can be regarded as a very difficult problem in the presence of noisy data, typically measured in coronary care units (CCU) or in emergency and ambulatory monitoring. Improvement of the detection performance in these conditions has been an important research line undertaken in our laboratory. Recently, a new approach that exploits the complementary information extracted from different sensors has been proposed [10]. 
The higher abstraction level takes advantage of the information obtained from the low-level and integrates it with medical knowledge, in order to propose an interpretation of the observed phenomena.

Three key aspects have to be addressed for such model-based ECG interpretation:

- Interfacing clinical observations with the model: Once the events of interest have been detected from the acquired signals, this stage calculates an error function indicating the likelihood of a given diagnostic hypothesis (represented as a set of model parameters) to explain the observation.

- Intra-beat model adaptation: Changes model parameters in order to reproduce and explain individual beats.

- Inter-beat model adaptation: Adjusts the parameters of the model so as to reproduce the observed cardiac rhythm (or a sequence of beats), by using parameters found during the intrabeat adaptation process.

This paper concentrates on the two first aspects. A preliminary inter-beat adaptation method has been presented elsewhere [7].

\section{OVERVIEW OF CARMEM}

CARMEM contains a dynamic, semi-quantitative model of the cardiac electrical activity, implemented in Simulink, using a cellular automata approach [9]. This model is inspired on a previous cardiac model $[19,20]$, developed in our laboratory during the 90's, and has been reengineered specifically for its integration into an on-line, model-based system. The main features of the CARMEM's model are:

- Sufficient physiological deep knowledge to simulate normal and major rhythm and conduction disorders,

- An abstraction level similar to that cardiologists use for cardiac arrhythmia diagnosis,

- Direct physiological interpretation of its internal parameters,

- An open architecture facilitating the integration of new knowledge (or new models), 
- A meta-level allowing the generation of explicit explanations, as well as an approach to dynamically adapt its structure and internal parameters.

The model can be broken down into three different levels: $i)$ Structural level, ii) Heart conduction and ECG synthesis level and iii) Meta-level.

\subsection{STRUCTURAL LEVEL}

The heart is modeled as a set of macro-cellular structures of two kinds representing nodal and myocardial tissues respectively. The structures are implemented by means of two different cellular automata one nodal (NA) and one myocardial (MA). The basic physiological model presents the same abstraction level as EINTHOVEN or HOLMES and is composed of 16 automata (ten NA and six MA), representing the following cardiac structures:

- $\quad$ the sino-atrial node (SAN);

- the upper and lower parts of internodal atrial tracts (UANT and LANT), which, although historically questioned, can help to explain some atrial conduction abnormalities [17];

- $\quad$ the atrio-ventricular node (AVN);

- $\quad$ the upper and lower parts of the bundle of His (UH and LH);

- the upper and lower, left and right bundle branches (ULBB, URBB, LLBB and LRBB), which have been artificially segmented in this way in order to facilitate the simulation of some particular rhythms, presenting both antegrade and retrograde conductions (fusions);

- $\quad$ the upper and lower parts of both atria (UA and LA) and

- $\quad$ the upper and lower, left and right ventricles (ULV, URV, LLV and LRV).

New automata can be added and connected dynamically to the basic model in order to represent other structures, such as ectopic pacemakers, pathological pathways like accessory pathways, etc.

However, as in other models presenting the same anatomo-physiological level of detail, the model in CARMEM is not able to generate some particular rhythms like atrial or ventricular fibrillation or flutter. These particular rhythms can be characterized by means of specific low-level signal processing methods [21, 27].

At any given time, each automaton can be in one of four physiological states: slow diastolic depolarization $(S D D)$ (for NA) or idle (for MA), upstroke depolarization period (UDP), absolute refractory period $(A R P)$ and relative refractory period $(R R P)$. At the end of the UDP of a given 
automaton, an activation impulse is sent to all the neighboring structures. In each automaton, state transitions are triggered both from the inside and from outside. In the former case, a set of internal parameters, representing the time associated with each physiological state, govern the transitions. In the later, the automaton is externally activated by the depolarization of a neighboring structure. The hysteresis properties of the cardiac tissue, namely, the cycle rate dependency of refractory periods and the response to premature activations, have also been modeled in each automaton by means of simple mathematical models [9].

Nodal automata are characterized by 6 real-valued parameters: four parameters representing the duration of each one of the physiological phases (SDD, UDP, ARP and RRP), and two others corresponding to the slope (or sensibility) of the two relations describing the hysteresis properties of the cardiac tissue. Myocardial automata present five parameters: the same parameters as for NA, but without the duration of the SDD. All the parameters characterizing each automaton can be modified dynamically (manually and/or automatically) during a simulation. For example, a nodal automaton representing an ectopic foci, can be enabled or disabled during the simulation by changing its $S D D$ duration parameter (an SDD duration value defined in the model to infinity, will disable the NA).

A simplified piece-wise linear representation of the action potential (AP) of each structure is calculated during a simulation. Simplified AP's are also useful to automatically generate beat interpretations. The individual contribution of each MA to the vectorcardiogram (VCG) is calculated during the depolarization and repolarization periods of each myocardial structure by a set of VCG templates (see appendix 1).

\subsection{HEART CONDUCTION SYSTEM AND ECG GENERATION LEVEL}

Connections between structures are defined at this level, according to anatomical considerations for antegrade, retrograde and lateral activations. These connections, as well as those associated with additional structures inserted during a simulation, can also be modified dynamically.

The VCG is calculated, at each time-step, by the addition of the individual contributions of all myocardial structures. Since the electrical axis of the heart is considered as a dynamic parameter at this level, simulation of $Q R S$ complex morphology variations derived from patient movements and respiration are possible. The VCG obtained can be projected onto any chosen set of ECG leads by means of a transformation matrix, using the current value of the electrical axis of the heart. Appendix 1 
presents a brief description of the VCG/ECG synthesis process. More details can be found in [9, 14, $20]$.

\subsection{META-LEVEL}

This level allows interfacing of CARMEM to other physiological models (such as a model of autonomic baroreflex) that can interact with the cardiac model, or for the implementation of metaalgorithms, that adapt physiological parameters during dynamic cardiac rhythm simulation or arrhythmia interpretation.

The automatic explanation of each simulated beat is also performed in this level by means of a spatio-temporal representation of the cardiac conduction, known as ladder (or Lewis) diagrams [24]. Each nodal structure of the model is associated with its corresponding zone on the ladder diagram, according to anatomical considerations. In order to generate the diagram, the following process is applied:

Let $y_{u}^{i}$ and $y_{l}^{i}$ be the upper and lower boundaries of the zone representing nodal structure $i$ on the ladder diagram, $t_{0}^{i}$ its initial depolarization time and $U D P^{i}$ its duration of the depolarization period. All this information is obtained directly form the model parameters and from the current value of the simplified action potential of each nodal structure. A point is thus drawn on the diagram, for each active nodal structure, at the position $\left(t, y^{i}\right)$, where $t$ is the current time instant and $y^{i}$ is defined by:

$$
y^{i}=\left\{\begin{array}{l}
\frac{y_{l}^{i}-y_{u}^{i}}{U D P^{i}}\left(t-t_{0}^{i}\right)+y_{u}^{i}, \text { for antegrade activations and } \\
\frac{y_{u}^{i}-y_{l}^{i}}{U D P^{i}}\left(t-t_{0}^{i}\right)+y_{l}^{i}, \text { for retrograde activations }
\end{array}\right.
$$

The left and right bundle branches are represented individually, making it easier to identify, for example, the affected branch in a bundle branch block.

The meta-level is essential to perform model-based interpretation, allowing the definition of an interface between the model and clinical observations and a way to adapt the cardiac model in order to reproduce observed phenomena.

\section{INTERFACE BETWEEN THE MODEL AND THE OBSERVATION}

The first problem to solve is to develop an appropriate interface between the observations and the output of the model. In other words, how to find a representation of the observed activity $\left(X_{O}=\right.$ 
$\left.g\left(E C G_{O}\right)\right)$ which can be quantitatively compared with the activity generated by the model $\left(X_{S}=M(P)\right)$,

The proposed interface level is presented in Figure 2 and consists of different steps:

1 A set of acquired ECG signals is preprocessed in order to minimize the different types of noise and baseline wandering.

2 Detection of observed $P$-waves and $Q R S$ complexes is achieved by using data fusion techniques presented in $[8,10]$.

3 The $i$-th observed beat (to be reproduced) is extracted from observed signals by means of simple windowing:

$$
B_{i, j}^{O}(k)=\left[E C G_{O, j}\left(\tau_{i}^{A V_{O}}-M_{1}\right), \ldots, E C G_{O, j}\left(\tau_{i}^{A V_{O}}+M_{2}\right)\right]^{T}
$$

where $E C G_{O j}(k)$ is the sample at time $k$ of lead $j$ of the observed ECG. $\tau_{i}^{A V_{O}}$ represents the instant of occurrence of the $i$-th detected $Q R S$ and $\left(M_{1}+M_{2}\right)$ is the width of the window, centered at the $Q R S$ complex peak. This window may include a $P$-wave, appearing at instant $\tau_{i}^{A A O}$. The same process is applied to the synthesized signal, to construct the simulated beat, $B_{i, j}^{S}(k)$.

4 A transformation is applied to $B_{i, j}^{O}(k)$ in order to obtain the same dynamic range as the simulated beat. Also, an estimation of the electrical axis of the heart is calculated from the observed signals.

5 The model adaptation is performed. It calculates an error function between $X_{O, i}=\left\{B_{i, j}^{O}(k), \quad \tau_{i}^{A V_{O}}, \quad \tau_{i}^{A A_{O}}\right\}$ and $X_{S, \dot{i}}=\left\{B_{i, j}^{S}(k), \quad \tau_{i}^{A V_{S}}, \quad \tau_{i}^{A A_{S}}\right\}$ which depends on the chosen intra-beat adaptation method. $B_{i, j}^{S}(k)$ represents the $i$-th synthesized beat for lead $j$ and $\tau_{i}^{A V_{S}}, \tau_{i}^{A A_{S}}$, the instants of appearance of the simulated $Q R S$ complex and $P$-wave, respectively. The best estimated solution (i.e. the set of model parameters that reproduce at best the observed beat) is retained for each beat and listed with its ladder diagram, which is generated automatically by the 
model. Parameters contained in this list of intra-beat characterization are used for the explanation of the whole observed rhythm.

\section{INSERT Figure 2 HERE}

Detected $P$-waves that are not contained in one of the $B_{i, m}^{O}(k)$ windows are considered as blocked $P$-waves and initiate a specific adaptation process, based on the alignment of the simulated and observed $P$-waves.

\section{INTRA-BEAT ADAPTATION}

Once the interface between observations and model output has been defined, interpretation of incoming beats becomes the search of the parameter set of each modeled cardiac structure that best reproduce the observed beat. Intra-beat adaptation can thus be seen as an optimization problem consisting of minimizing, for each beat $i$, the error function of the difference between synthesized and observed activity. This error function can be expressed as:

$$
\varepsilon_{i}\left(X_{O, i}, X_{S, i}\right)=\varepsilon_{i}\left(X_{O, i}, M\left(P_{i}\right)\right)
$$

Since the model parameters convey a direct physiological interpretation, an explanation of the $i$-th observed beat can be obtained from the optimal set of parameters, $P_{i}^{*}$, that minimizes this error function. Nevertheless, a number of obstacles have to be overcome in order to have a meaningful and practical optimization process, among them:

- Due to the semi-quantitative nature of the CARMEM model, the error function is not differentiable with respect to individual parameters of each modeled structure. This aspect prevents the use of deterministic optimization algorithms, such as those based on gradient descent.

- The model depends on a large number of variables. 
- The parameter adaptation process, designed to reproduce the observed ECG, is an ill-posed problem, comparable, in a minor scale, to the inverse problem of the electrocardiography.

These difficulties make it mandatory the use of an optimization technique which is: $i$ ) independent of the existence of the derivative of the error function with respect to the model's parameters (i.e. the search of solutions has to be based solely on the evaluation of the error function), ii) adapted to complex problems including a great number of parameters and multiple local optima, and iii) robust. Two types of optimization methods seem adequate to this problem: exhaustive search and combinatory or stochastic search methods.

The first group was not considered here, due to their computational cost. In the second group, two methods were tested in an initial phase: simulated annealing [13] and evolutionary algorithms (EA) [11],[15]. Evolutionary algorithms have been the subject of intensive research during the last decade and have shown to be useful in the solution of hard identification problems, including different biomedical applications [18]. In EA's, the additional property of evaluating many potential solutions at the same time is particularly adapted to our problem, because it allows the definition of a set of predefined beats, which are commonly observed in clinical practice, as initial solutions. Due to these facts, EA's were kept as the identification approach for the adaptation process.

\subsection{INTRA-BEAT ADAPTATION BY EVOLUTIONARY ALGORITHMS}

Figure 3 depicts graphically the application of EA's for intra-beat adaptation. Convergence and robustness properties of EA's have been largely studied in the literature $[1,3,5]$. These properties depend upon: $i$ ) adequate individual coding, $i$ ) proper definition of the fitness function used for the evaluation of individuals and iii) selection of appropriate genetic operators for cross-over and mutation. These aspects are discussed in the following paragraphs. Modifications undertaken on classic evolutionary methods to integrate the physiological knowledge are also described.

INSERT Figure 3 HERE. 


\subsubsection{INDIVIDUAL REPRESENTATION}

Each individual of the EA represents an instance of the whole model of CARMEM. The chromosome of each individual can be seen as the concatenation of 21 segments, representing the 16 fundamental structures of CARMEM, 4 nodal structures, representing ectopic foci connected to each modeled ventricular structure and one myocardial structure, characterizing a right free wall accessory pathway. Segments of nodal structures are constituted of three real values, with temporal parameters for the $S D D$, the $U D P$ and the $A R P$. Segments associated with myocardial structures present only two parameters, $U D P$ and $A R P$. The two parameters of nodal and myocardial structures specifying the sensibility to the hysteresis properties of the cardiac tissue, and the relative refractory period of all structures, are assumed constant during the adaptation process and defined to their specific default values. Thus, chromosomes are composed of $G=56$ real values as shown in Figure 4. Chromosome definition is accompanied by a matrix indicating the possible upper and lower limits for each parameter (Figure 4). This matrix is employed by the EA, in order to synthesize physiologically feasible beats, during the construction of the initial population and the application of genetic operators.

\section{INSERT Figure 4 HERE.}

\subsubsection{INITIALIZATION OF THE POPULATION}

The initial population is constituted of 16 predefined individuals and 34 variations. Predefined individuals represent beats from different origins (sino-atrial node, atrio-ventricular $(\mathrm{A}-\mathrm{V})$ node and ectopic foci connected to the ventricles) and conduction disturbances (such as A-V blocks, branch blocks,...). Variations are built by random selection, with replication, of one of the 16 predefined individuals and by modifying, also randomly, one of its genes in the valid interval. Moreover, for the adaptation of beats $i>1$, the solution of the beat $i-1$ is also included in the initial population.

This initialization technique contributes to a faster convergence and an improved robustness, avoiding premature convergence to a local minimum. It also allows integration of explicit medical knowledge, by introducing as individuals a set of beats observed often in clinical practice. 


\subsubsection{ERROR FUNCTION AND SELECTION METHOD}

The interface between the model and the observations, defined in section 3 , is used here to calculate the performance of each individual $l$, by means of the error function $\varepsilon\left(X_{O}, X_{S}\right)$. Three sources of error between the synthesized and the observed activities can be identified to construct the error function $\varepsilon$ :

1. The sample-to-sample morphological difference between the observed and synthesized beat:

$$
e_{M}(l, k)=\left|B_{i, j}^{O}(k)-B_{i, j}^{S l}(k)\right|, k=1, \ldots, M_{B}
$$

where $B_{i, j}^{o}$ represents lead $j$ of the observed beat, $B_{i, j}^{s, l}$ is the synthesized beat for the same lead $j$ by individual $l . M_{B}$ is the size of the evaluation window $\left(M 1+M 2=M_{B}=800 \mathrm{~ms}\right)$.

2. The difference between occurrence instants in the observed and synthesized $P$-waves $\left(\tau_{i}^{A A_{O}}\right.$ and $\tau_{i}^{A A S}$ respectively) for individual $l$, obtained as:

$$
e_{A A}(l)=\left|\tau_{i}^{A A_{O}}-\tau_{i, l}^{A A_{S}}\right|
$$

3. The difference between observed $\left(\tau_{i}^{A V_{O}}\right)$ and synthesized $\left(\tau_{i}^{A V_{S}}\right) Q R S$-complex timing, which is always zero, due to the application of the procedure of $Q R S$ alignment in the interface algorithm.

Thus, the error function for individual $l$ and beat $i$ can be calculated by:

$$
F_{l}=\sum_{k=1}^{M_{B}}\left(e_{M}(l, k)\right)+\alpha \cdot e_{A A}(l)
$$

Where constant $\alpha \in[0,1]$, allows to establish the relative weight of the error associated with the generation of the $P$-wave.

Once the error function has been evaluated for each individual, selection based on the ranking method, adapted for function minimization, is carried out [15]. All the information obtained from the model for each individual $l$ (i.e. time of occurrence of simulated $P$-waves, simulated action potentials for all structures and the ladder diagram) is saved for future use during cross-over and mutation operations. 


\subsubsection{KNOWLEDGE INTEGRATION INTO GENETIC OPERATORS}

Convergence improvement and reduction of computational load of an evolutionary algorithm can be achieved by means of different evolution strategies $[1,3,4]$. One of them consists in determining, for a given individual $l$, two important values, in order to improve its performance: $i$ ) the gene, or set of genes, that should be modified to improve the solution and ii) the direction of this modification, whether positive or negative. This information can be compared to the error function gradient with respect to each parameter. Normally, no information on the gradient is employed in genetic operators. Both, the gene to modify and the direction of the modification are obtained randomly following a given distribution. In the case of the intra-beat adaptation, some electrophysiological knowledge can be used in order to give a higher modification probability to specific genes. Particularly, three heuristic criteria have been used to improve the adaptation of the EA:

1. ECG wave duration is mainly controlled by the rapid depolarization period (UDP) of cardiac structures. For example, a long $Q R S$ complex, observed in a bundle branch block, is associated with a conduction delay in this structure. This first criterion $\left(w_{B}\right)$, that attributes a more important weight to genes $(q)$ representing the $U D P$ period, is defined by:

$$
w_{B}(q, l)=\left\{\begin{array}{cc}
1 & \text { if } \\
0.5 & q \text { represents the UDP }
\end{array}\right.
$$

2. The morphological error $e_{M}(l, k)$ of a specific part of the beat can be related to a set of cardiac structures. For example, the morphological error of the $Q R S$ complex is related to ventricular structures. This second criterion $\left(w_{M}\right)$ assigns a higher weight to genes deriving a higher morphological error $e_{M}(l, k)$. This information is extracted from action potentials generated by the model during the simulation. The value of the second criterion is the same for all genes $q$ of a given cardiac structure $S$ and is given by:

$$
w_{M}(q, l)=\sum_{k=M 1_{S}}^{M 2 S} e_{M}(l, k), \quad \forall q \in S
$$


Values $M 1_{S}$ and $M 2_{S}$ represent respectively the activation instant (start of the $U D P$ ) and the end of the $R R P$ period, detected from the action potential of structure $S$.

3. The final criterion is based upon the fact that the difference between the observed and simulated times of occurrence of $P$-waves (i.e. the error $\left.e_{A A}(l)\right)$ can be controlled by modifying the UDP of atrio-ventricular nodal structures (i.e. the A-V node, upper and lower bundle of His, etc...). This criterion $\left(w_{A A}\right)$ can be calculated only if the occurrence time for the observed $P$-wave is available. It assigns a higher weight to genes representing the UDP of A-V structures, by:

$$
w_{A A}(q, l)=\left\{\begin{array}{cc}
\beta \cdot e_{A A}(l) & \text { if } q \text { represents the } U D P \text { of a junctional structure } \\
0 & \text { else }
\end{array}\right.
$$

and $\beta \in[0,1]$.

These criteria have been included in the EA, by calculating or assigning a weight, $w(q, l)$, to represent the modification likelihood for gene $q$ of individual $l$ during mutation and cross-over. The final selection weight for gene $q$ of individual $l$ is given by:

$$
w(q, l)=w_{B}(q, l)+w_{M}(q, l)+w_{A A}(q, l), q=1, \ldots, G
$$

The developed EA is thus driven by a mutation process (geometrical creep mutation) and by two cross-over methods (uniform and heuristic cross-over) [1]. The random function generator used for these operators has been modified in order to take advantage of the proposed criteria $(w(q, l))$, giving higher modification probabilities to the genes with higher weights.

\subsection{EVALUATION OF THE ROBUSTNESS OF THE INTRA-BEAT ADAPTATION}

As mentioned earlier, general robustness properties of EAs have been largely studied in the literature $[4,5]$. However, in order to evaluate the effectiveness of the customized genetic operators and 
the variability of the proposed beat interpretations, a robustness analysis has been designed. It is based on the following steps:

1. 35 realizations of the intra-beat evolutionary algorithm were applied to each one of the beats selected from the MIT-BIH arrhythmia database [16]. Each realization of the EA was stopped when the error of the best individual of the population was lower than a predefined threshold.

2. For each realization of the intra-beat EA, the following data were saved for further analysis: $i$ ) the set of parameters of the best individual of the last generation, $i i$ ) its ladder diagram, iii) the error value of the best individual and iii) the EA computation time.

3. Beat interpretations (ladder diagrams) obtained from the solutions of each realization of the EA were compared between them, and to the annotations of the MIT-BIH database, with the aid of a specialist.

4. The global conduction times through the atria, the atrio-ventricular junction and the left and right bundle branches were calculated for each one of the 35 realizations, by adding the corresponding model parameters (i.e. the conduction time through the atria equals the sum of the UDP parameters of the UANT and LANT structures). These values were analyzed by means of boxplots [22], in order to better quantify the variability of the proposed solutions.

\section{RESULTS}

The first evaluation stage of the intra-beat adaptation process required several tests reproducing five different beat morphologies, associated with distinct pathologies, in order to find appropriate parameter values for the EA. The best results were obtained with a relatively high mutation probability $p_{m}$ (close to 0.6 ) and a cross-over probability $p_{r}$ close to 0.3 , corresponding to a "naïve evolution" strategy, which mimics the evolution of an "asexual" population and has been used successfully on a number of applications [1]. The size of the population has been fixed to 50 individuals. The stopping criterion is passed if no solution improvement is found during 15 consecutive generations, or if the maximum number of iterations (fixed to 400) is reached. Parameters $M_{l}$ and $M_{2}$ of the interface definition (equation 2) have been set to 300 and $500 \mathrm{~ms}$ respectively. Results are calculated by using the most 
representative ECG lead for the studied pathology, chosen from the two leads available from the MITBIH arrhythmia database.

Three examples of intra-beat adaptation are presented in this section: the first one is applied to a sinus beat with normal $\mathrm{A}-\mathrm{V}$ conduction, the second to a left branch block and a third to a premature ventricular contraction (PVC). Beats presented in the first and third examples have been extracted from record 100 on the MIT-BIH arrhythmia database. The second example shows a beat taken from record 111 of the same database. In each case, the explanation generated by the model has been compared to the annotations of the database, with the aid of a specialist. Figures in this section show intra-beat adaptation examples, presenting the best individual of the initial population with its ladder diagram. Some of the best individuals of later generations are also presented, in order to show the different hypotheses that are generated during the evolution of the EA. Finally, section 5.1.4 is dedicated to the robustness of the adaptation process.

\subsubsection{EXAMPLE 1: SINUS BEAT WITH NORMAL A-V CONDUCTION (FIGURE 5)}

The best individual of the initial population (generation 0) is a sinus beat with a large energy $Q R S$ and a displaced $P$-wave in relation to the observation (Figure 5). In generation 9 , the instant of appearance of the simulated $P$-wave is closer to the observed one, while $Q R S$ amplitude was reduced. This tendency is maintained through the following generations (see, for example, generation 27). In generation 46, a new hypothesis is proposed by the EA: a slight conduction delay in the left bundle branch that could explain the delayed rising front of the observed $R$-wave. This hypothesis remains as the best individual until convergence, in generation 74, when $Q R S$ morphology and $P$-wave timing are very close to the observation. The biphasic $T$-wave in the observed beat was not accurately synthesized due to the limitations of CARMEM's $T$-wave templates. 


\subsubsection{EXAMPLE 2: LEFT BUNDLE BRANCH BLOCK (FIGURE 6)}

Figure 6 depicts intra-beat adaptation algorithm generations for a case of left bundle branch block. In the beginning of the adaptation process (between generations 0 and 2), the best adapted individual presents an active right free wall accessory pathway (which accounts to the energy found at the beginning of the $Q R S$, or delta wave) and a slightly delayed ventricular conduction. The hypothesis of left bundle branch block is started after generation 3 (see ladder diagram of generations 3 to 73 ). The hypothesis of right bundle branch block, present in the initial population, is later rejected because peak amplitude, $\mathrm{R}$ and $\mathrm{R}^{\prime}$, are inverted with respect to the observation. From the third generation on, the adaptation process seeks to improve $P$-wave timing and $Q R S$ complex morphology. The final solution, shows important conduction delays in junctional structures (characteristic of a first-degree A-V block) and an important conduction delay at the left bundle branch (left bundle branch block). This solution is consistent with the annotation on the database.

INSERT Figure 6 HERE

\subsubsection{EXAMPLE 3: PREMATURE VENTRICULAR CONTRACTION (FIGURE 7)}

In this example (Figure 7), the best individual of the initial population represents an ectopic beat of ventricular origin, initiated at the inferior left ventricle with retrograde conduction to the atria. This solution is also confirmed by the annotation of the database. The error is slightly reduced during the following generations and, at generation 14th, an appropriate explanation of the beat is available. Nevertheless, as mentioned before, CARMEM was unable to accurately model observed $T$-waves. This leads to a situation in which later generations tend to eliminate the valid solution in order to cope with $T$-wave morphological errors, which presents an important energy. In the 32 nd generation, a new hypothesis is found where the synthesized $T$-wave is closer to the observation. This solution exhibits a first ectopic activation, starting at the left ventricle, with retrograde conduction through the left bundle branch, through the His bundle and finally to the atria. At the same time, the impulse is transmitted to the right bundle branch, showing an antegrade activation of the right ventricle to produce a positive $T$ wave. A new explanation is obtained in the 39th generation, where two impulses are simultaneously 
generated (one of junctional origin and another from the left ventricle). The junctional activation presents a retrograde conduction to the atria and an antegrade conduction to the ventricles. This antegrade activation does not attain the depolarization of the left ventricle that has been previously activated by an ectopic focus. This example shows how a beat explanation can diverge to unlikely solutions in order to minimize morphological error.

One interesting element of these results can be observed between generations 14th and 32nd, where a negative $P$-wave generated by the model (represented by $*$ and produced by the retrograde activation of the atria), coincides with that of the observed beat (represented by $\square$ ). It is possible to see how it is feasible to take advantage of knowledge within the model to generate valid hypotheses on wave locations, especially the $P$-waves, which are often very difficult to detect when they are embedded into the $Q R S$ complex.

\section{INSERT Figure 7 HERE}

\subsubsection{RESULTS OF THE ROBUSTNESS TEST}

Figure 8 presents four different solutions, which are representative of those obtained from 35 realizations of the intra-beat adaptation, applied to the beat shown in example 2 (section 5.1.2). It can be seen that, even if the ladder diagrams differ slightly, the interpretation proposed by these different solutions remains coherent. For example, the A-V delay of the observed beat can be explained by a very slow conduction at the AV node and an almost normal conduction through the his bundle, (Figure $8 a$ ), or equivalently slow conductions through the AV node and the his bundle (Figure $8 b$ to $d$ ), the conduction time between the atria and the ventricles remaining comparable. Some differences of the same nature can also be observed in the right bundle branch (see, for example, Figure $8 c$ and $d$ )

In order to statistically quantify this variability, boxplots of these results are shown in Figure $8 e$, in which the corresponding values of the four solutions ( $a$ to $d$ ) are identified with different symbols (see [22] for a description of boxplots). Solutions of all realizations provide similar values of global conduction times, confirming the robustness of the interpretation for this beat. The lowest variability (more compact boxplot -and more reliable identification) was observed for the left bundle branch conduction time, which is a key parameter for the identified pathology. Wider dispersions are observed 
for the identification of the conduction times through the AV junction and right bundle branch. Although the atrial conduction time presents a compact boxplot, 6 outlier solutions can be observed ('+' symbols), making it the least reliable marker. Two of these outliers, marked with the symbols $\mathrm{O}$ and $\square$, are shown in Figure $8 b$ and $c$. This can be explained in part by the low amplitude of the observed $P$-wave.

\section{INSERT Figure 8 HERE}

\section{DISCUSSION}

During the initial evaluation of the EA, the best results were found by adopting a "naïve evolution" strategy. Two main reasons led us to retain this approach: $i$ ) the specific physiological knowledge allowing us to improve the EA's convergence, represented by means of customized genetic operators (section 4.1.4), is more effective for mutation than for crossover and ii) a high crossover probability led to a big number of aberrant solutions during the initial generations, containing, for example, two or more active ectopic foci. In the current EA implementation, values for $p_{r}$ and $p_{m}$ remain constant throughout the whole adaptation process. A specific rule can be defined to modify these probabilities in order to accelerate convergence.

Robustness analysis of supra-ventricular beats, with or without conduction disorders, presented similar results to those of section 5.1.4. For ectopic beats of ventricular origin, the pacemaker structure of the proposed solution stayed coherent for different realizations, but showed important differences in the conduction path (such as those shown in Figure 7). These explanations represent local minima of the error function, leading always to good morphological reproductions of the observed beat. Most of the convergence errors observed for the intra-beat adaptation are related to the reproduction of PVC's and are a consequence of the $T$-wave synthesis limitations.

The calculation time for intra-beat adaptation depends, obviously, on the number of generations necessary to obtain population convergence, which depends itself on the difference between the observed beat and the individuals of the initial population. Mean computation time of the intra-beat EA, obtained from the robustness analysis of section 5.1.4, was of 19 minutes, running on a PowerMac G4 at $733 \mathrm{MHz}$ and starting from the initial population described in section 4.1.2. For subsequent beats 
of the same rhythm, the best solution of previous beats is saved in the initial population, reducing significantly the calculation time (around 40 seconds for the second beat). It should be noted that the whole system has been implemented in Matlab/Simulink (which is an interpreted language running slower than a compiled one) and that both the cellular automata model and the evolutionnary algorithm used are inherently parallelizable.

Another interesting point concerns the potential utilization of the model as the source of knowledge in cardiac event detection, particularly for $P$ waves. Indeed, if the occurrence time of observed $P$-waves is not introduced as parameter to the intra-beat adaptation algorithm (case of a miss-detection at the low level), only the morphological differences are used in the error function. Although this implies a slower convergence, if the observed $P$-wave presents sufficient energy, the EA is capable of finding its position by minimizing the morphological error. Moreover, in cases where the $P$-wave is not easy to detect, as in the example 3, the implicit knowledge of the cardiac conduction system represented in the model allows us to produce a hypothesis about its location.

\section{CONCLUSIONS}

This work has demonstrated the feasibility of using deep physiological model-based interpretation of the cardiac rhythm, combined with a direct interface to real observations. The proposed approach differs considerably from manually interfaced cardiac rhythm analysis model-based systems. Two key aspects were developed: model-observation interfacing and intra-beat adaptation.

Model-observation interfacing relies on robust detection algorithms for $P$-waves and $Q R S$ complexes proposed in our laboratory. Another original characteristic of the interface resides in the fact that the main element of comparison between patient observation and simulated activity is based on the recorded ECG signal. To the best of our knowledge, CARMEM is the only model-based system providing such integration.

Intra-beat adaptation was approached as an optimization problem, requiring the implementation of stochastic search algorithms. The proposed approach, based on evolutionary methods, differs notably from those of current model based systems, which are often implemented using a set of first-order logic rules. Physiological knowledge has been integrated in individual representation, in the control of genetic operators (mutation and cross-over) and in the calculation of the error function. Population 
initialization is based upon a set of hypotheses (beat explanations) observed commonly in clinical practice, leading to an improved robustness and convergence speed of the adaptation process.

Preliminary adaptation results are encouraging and show how the anatomic and physiological knowledge can be exploited in order to explain underlying disorders, to better localize their origin, to retrieve missed detections and to correct false alarms obtained from the signal processing stage. In the long run, a similar model-based approach could also be used to explain differences between predicted patient's states and observations.

Some limitations still persist in the model and they represent the motivations for current and future development. The most important one concerns the ECG synthesis stage. Although the templates used are useful in generating beats with antegrade activations, this is not the case for retrograde conductions, limiting the capability of the model to reproduce and explain these phenomena. Development of ECG synthesis based on the equivalent source method is a possibility to solve the problem, however, it will increase considerably computational costs.

Finally, an inter-beat adaptation stage, aiming at reproducing the global activity of an observed rhythm, has to be developed. This is a complex task that has to take into account, on one hand, the individual solutions of the intra-beat adaptation (explanation of individual beats) and, on the other, the preservation of the global physiological state and the continuity of parameter values between consecutive beats (rhythm explanation). 
Due to the semi-quantitative nature of the CARMEM's model and in order to optimize computational costs during model simulation and adaptation, a particular VCG and ECG synthesis stage had to be designed. It is based on the same principles, already described in a previous version of our model $[14,20]$. This short appendix recalls the fundamental aspects of the synthesis stage, which is performed, for each time instant, in four different steps:

- The electrical vector produced by each myocardial structure is calculated, with respect to a predefined heart-related axis.

- A global activation vector is calculated by the sum of the individual contributions of all active myocardial structures.

- The global activation vector is projected from the heart-related axis, into a body-related axis in order to obtain the VCG.

- $\quad$ The VCG is projected into a set of ECG leads.

The contribution of each myocardial structure to the surface ECG depends on: 1) its current electrical state, 2) its volume, 3) the propagation direction inside the structure, 4) its anatomical position in the myocardium and 5) the duration of its current electrical phase. Each myocardial structure generates a particular time-varying electrical vector, depending on these conditions. The set of all these electrical vectors constitutes the template database used by the ECG synthesis stage.

\subsection{TEMPLATE DEFINITION}

Each MA contains templates representing its contribution to the $\mathrm{X}, \mathrm{Y}$ and $\mathrm{Z}$ axes of the VCG, for different electrophysiological conditions. For example, Figure 9 shows the Simulink diagram of the base cardiac model of CARMEM and the VCG contribution templates of each MA in the case of a normal, antegrade activation. These templates are defined with respect to a heart-related local axis. Templates have been defined for the atria and the ventricles.

Atrial templates: Two different cases were considered for both the upper and lower atria: antegrade and retrograde depolarization. As atrial repolarization is never perceptible on real ECGs, it has not been taken into account in the current version of the model. 
Ventricular templates: The direction and the general form of ventricular depolarization vectors depend closely on the activation origin. For example, 5 different cases have been considered for the upper part of the right ventricle:

- normal antegrade activation from the bundle branch,

- $\quad$ retrograde depolarization from the bundle branch;

- $\quad$ retrograde, muscle-to-muscle depolarization from the lower part of the right ventricle;

- $\quad$ antegrade depolarization coming from the accessory pathway;

- depolarization from an ectopic foci, located in the upper part of the right ventricle.

For the ventricular repolarization phase, only three cases were considered, corresponding to antegrade conductions, retrograde conductions and antegrade repolarization after an activation from the accessory pathway. Only the right free wall location of accessory pathways has been considered in the current version.

Synthetically, for the six myocardial structures and the different activation directions (i.e. antegrade, retrograde, lateral, ...), 30 different template vectors have been defined, that is, a total of 90 different predefined waveforms. The determination of these templates has been made in an iterative manner, by successive approximations, guided by a set of ECG and VCG observations, corresponding to selected normal and pathological cases. Three steps were applied iteratively: $i$ ) extraction of ECG invariants; $i$ ) projection of the observed X-Y-Z axes, to a body-related axis, $\boldsymbol{M}_{c, b}$, and to a heart-related axis, $\boldsymbol{M}_{b-d}$, based on the observed electrical axis of the heart; iii) qualitative determination of the individual contribution of each structure and $i v$ ) validation. This work, which can be compared with the determination of wave morphology rules of previous model-based systems, is described in detail in $[14]$.

\subsection{VCG AND ECG SYNTHESIS}

The generation of the VCG implies: $i$ ) the sum of the individual contribution of all active MA and ii) the projection from a heart-related axis $V C G_{c}(k)=\left[\begin{array}{llll}X_{c}(k) & Y_{c}(k) & Z_{c}(k)\end{array}\right]^{T}$ to an axis defined with respect to the body of the patient $V C G_{b}(k)=\mathbf{M}_{c, b} \cdot V C G_{c}$, by means of the following transformation:

$$
\mathbf{M}_{c, b}=\left[\begin{array}{ccc}
\cos \alpha & \sin \alpha & 0 \\
-\sin \alpha & \cos \alpha & 0 \\
0 & 0 & 1
\end{array}\right]
$$


where the value $\alpha$ represents the patient's heart electrical axis.

The 12 standard ECG leads can be calculated by using a projection defined by: and the relation $E C G_{\text {lead }}(k)=\mathbf{M}_{b, d} \cdot V C G_{b}(k)$. Each ECG lead is built by using a specific couple of values for $\theta$ and $\varphi$. 


\section{References}

[1] Beasley, D., Bull, D. R., Martin, R., An Overview of genetic algorithms: Part 2, Research Topics, University Computing. 15, (1993) 170-181.

[2] Bratko, I., Mozetic, I., Lavrac, N., KARDIO: A study in deep and qualitative knowledge for expert systems. (The MIT Press, Cambridge, MA, 1989).

[3] Davis, L., Genetic Algorithms and Simulated Annealing. (Pitman Publisher, New York, 1987).

[4] Davis, L., Handbook of Genetic Algorithms. (Van Nostrand Reinhold, New York, 1991).

[5] Goldberg, D. E., Genetic Algorithms in search, optimization and machine learning. (Addison-Wesley, Boston, MA, 1989).

[6] Guertin, M., Abductive inference of events: diagnosing cardiac arrhythmias, Phd Thesis Boston University (1996).

[7] Hernández, A., Fusion de signaux et de modèles pour la caractérisation d'arythmies cardiaques, Phd Thesis Université de Rennes 1 (2000).

[8] Hernández, A. I., Carrault, G., Mora, F., Improvement of a P-wave detector by a bivariate classification stage, Transactions of the institute of measurement and control. 22, (2000) $231-242$.

[9] Hernández, A. I., Carrault, G., Mora, F., Bardou, A., Overview of CARMEM: A new dynamic quantitative cardiac model for ECG monitoring and its adaptation to observed signals, Acta Biotheoretica. 48, (2000) 303-322.

[10] Hernández, A. I., Carrault, G., Mora, F., Thoraval, L., Passariello, G., Schleich, J. M., Multisensor fusion for atrial and ventricular activity detection in coronary care monitoring, IEEE Transactions on biomedical engineering. 46, (1999) 1186-1190.

[11] Holland, J. H., Adaptation in Natural and Artificial Systems. (MIT Press, MA, 1975).

[12] Hunter, J., Kirby, I., Gotts, N., Using Quantitative and Qualitative Constraints in Models of Cardiac Electrophysiology, Artificial Intelligence in Medicine. 3, (1991) 41-61.

[13] Kirkpatrick, S., Gelatt, C. D., Vecchi, M. P., Optimization by simulated annealing, Science. 220, (1983) 671-680. 
Le Moulec, F., Etude et réalisation d'un modèle qualitatif profond de l'activité électrique du coeur pour un système de monitoring intelligent en unité de soins intensifs pour coronariens, Phd Thesis Université de Rennes 1 (1991). Cardiol. 78, (1996) 927-31.

Zhang, X. S., Zhu, Y. S., Thakor, N. V., Wang, Z. Z., Detecting ventricular tachycardia and fibrillation by complexity measure, IEEE Transactions On Biomedical Engineering. 46, (1999) 548-55 


\section{Figure Captions}

Figure 1. Abstraction levels of an intelligent cardiac monitoring system. Segmented arrows represent possible relations from the high to the low abstraction levels that could be developed in the future, for example, to assist the ECG wave detection algorithms (see example 3).

Figure 2. Diagram of the proposed interface level.

Figure 3. General diagram of the evolutionary algorithm used for intra-beat adaptation.

Figure 4. Chromosome representation for the evolutionary algorithm of intra-beat adaptation.

Figure 5. Model adaptation to a sinus beat in record 100 of the MIT-BIH database. Each panel shows the best individual of some populations of the EA, presenting the observed beat (thicker line) and the synthesized beat (finer line) along with its ladder diagram.

Figure 6. Adaptation of the model to a beat presenting a left bundle branch block obtained from record 111 of the MIT-BIH database.

Figure 7. Application of the intra-beat adaptation algorithm to a premature ventricular contraction in record 100 of the MIT-BIH database.

Figure 8. Panels $a$ to $d$ : Four solutions obtained from different realizations of the intra-beat adaptation algorithm for the same observed beat. Panel $e$ : Boxplot of conduction times through the atria, the atrio-ventricular junction and the left and right bundle branches, calculated from 35 different realizations of the intra-beat adaptation algorithm. The upper and lower parts of each box represent the last and the first quartile of the population, respectively; the line at the middle of the box represents the median value; the ' + ' symbol represents the outliers; the lines showing upper and lower limits symbolize the extent of the observed samples (without the outliers) and the notches show the $95 \%$ confidence interval of the mean value. Symbols $\downarrow, \mathrm{O}, \square$, and $\triangle$ show, respectively, the position of solutions $a, b, c$ and $d$ on the boxplot.

Figure 9. Simulink diagram of CARMEM's model (normal case without ectopic foci or accessory pathways) presenting the templates of each MA for the case of a normal antegrade conduction. 


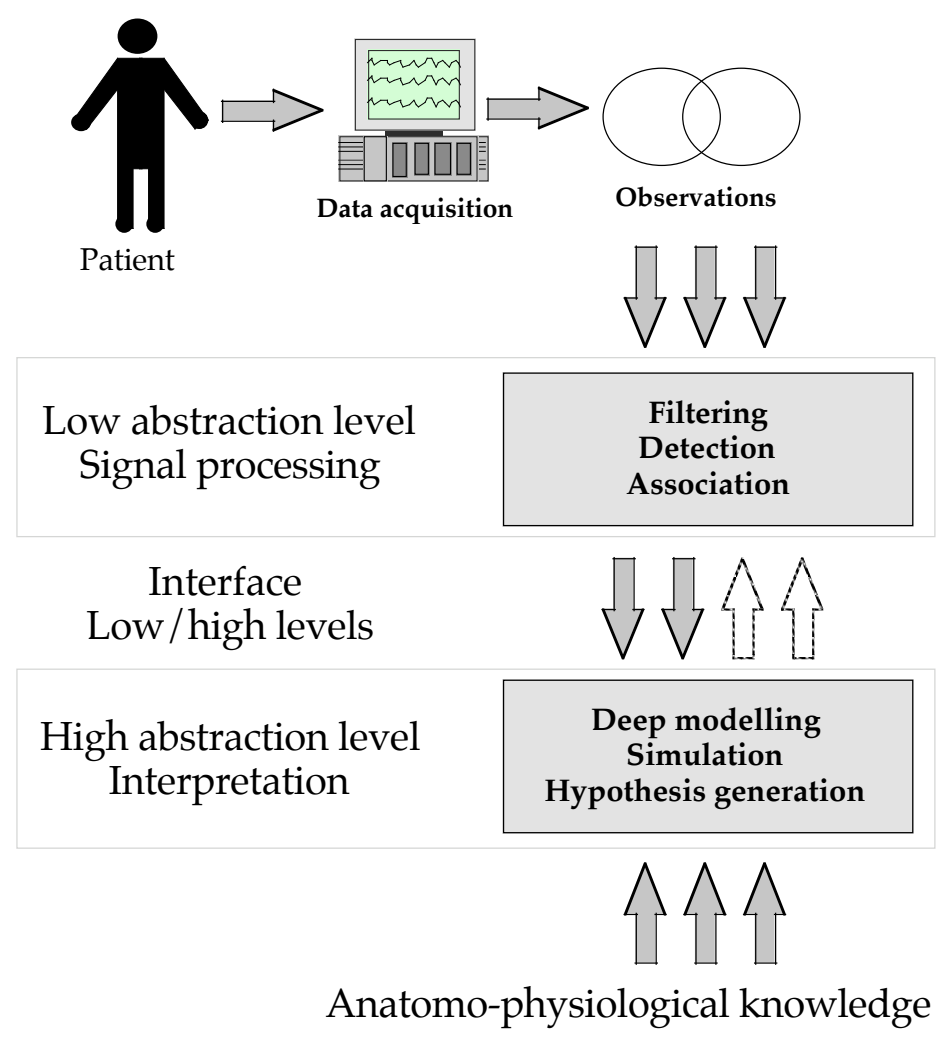

Figure 1. Abstraction levels of an intelligent cardiac monitoring system. Segmented arrows represent possible relations from the high to the low abstraction levels that could be developed in the future, for example, to assist the ECG wave detection algorithms (see example 3). 


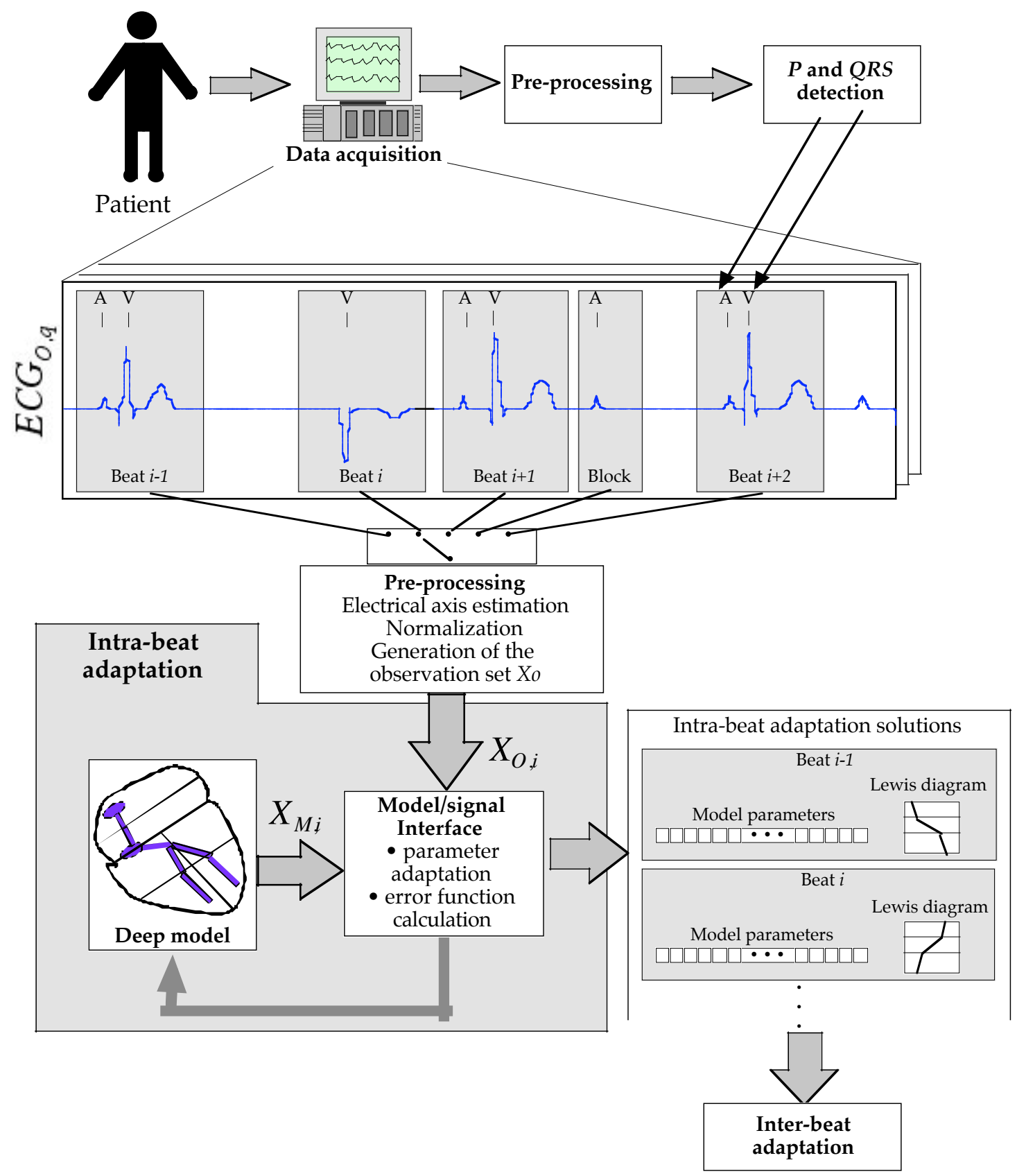

Figure 2. Diagram of the proposed interface level. 


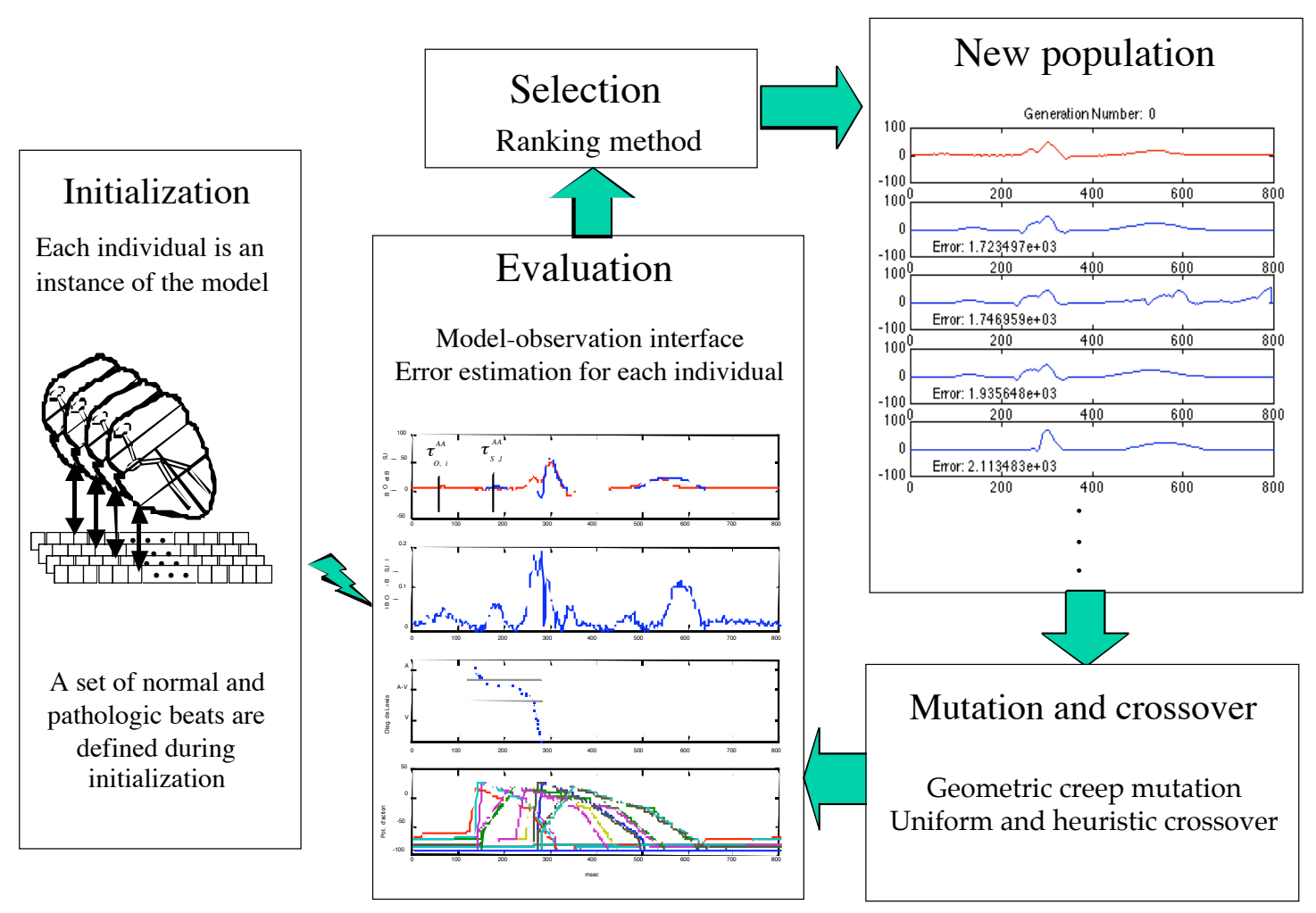

Figure 3. General diagram of the evolutionary algorithm used for intra-beat adaptation. 


\begin{tabular}{|c|c|c|c|c|}
\hline $\begin{array}{c}\begin{array}{c}\text { Segment of a } \\
\text { nodal structure }\end{array} \\
\end{array}$ & & $\begin{array}{l}\text { Segm } \\
\text { ocardi }\end{array}$ & $\begin{array}{l}\text { ent of a } \\
\text { al stru }\end{array}$ & \\
\hline$S D D_{1} U D P_{1} A R P_{1}$ & $\bullet \bullet$ & $U D P_{i}$ & $A R P_{i}$ & \\
\hline
\end{tabular}

\begin{tabular}{|c|c|c|c|c|c|c|}
\hline Minima & $S D D_{1}^{-} U D P_{1}^{-}$ & $A R P_{1}^{-}$ & $\bullet \bullet$ & $U D P_{i}^{-}$ & $A R P_{i}^{-}$ & $\bullet \bullet$ \\
\hline Иа & $S D D_{1}^{+} U D P_{1}^{+}$ & $A R P_{1}^{+}$ & $\bullet \bullet$ & $U D P_{i}^{+}$ & $A R P_{i}^{+}$ & $\bullet \bullet$ \\
\hline
\end{tabular}

Figure 4. Chromosome representation for the evolutionary algorithm of intra-beat adaptation. 


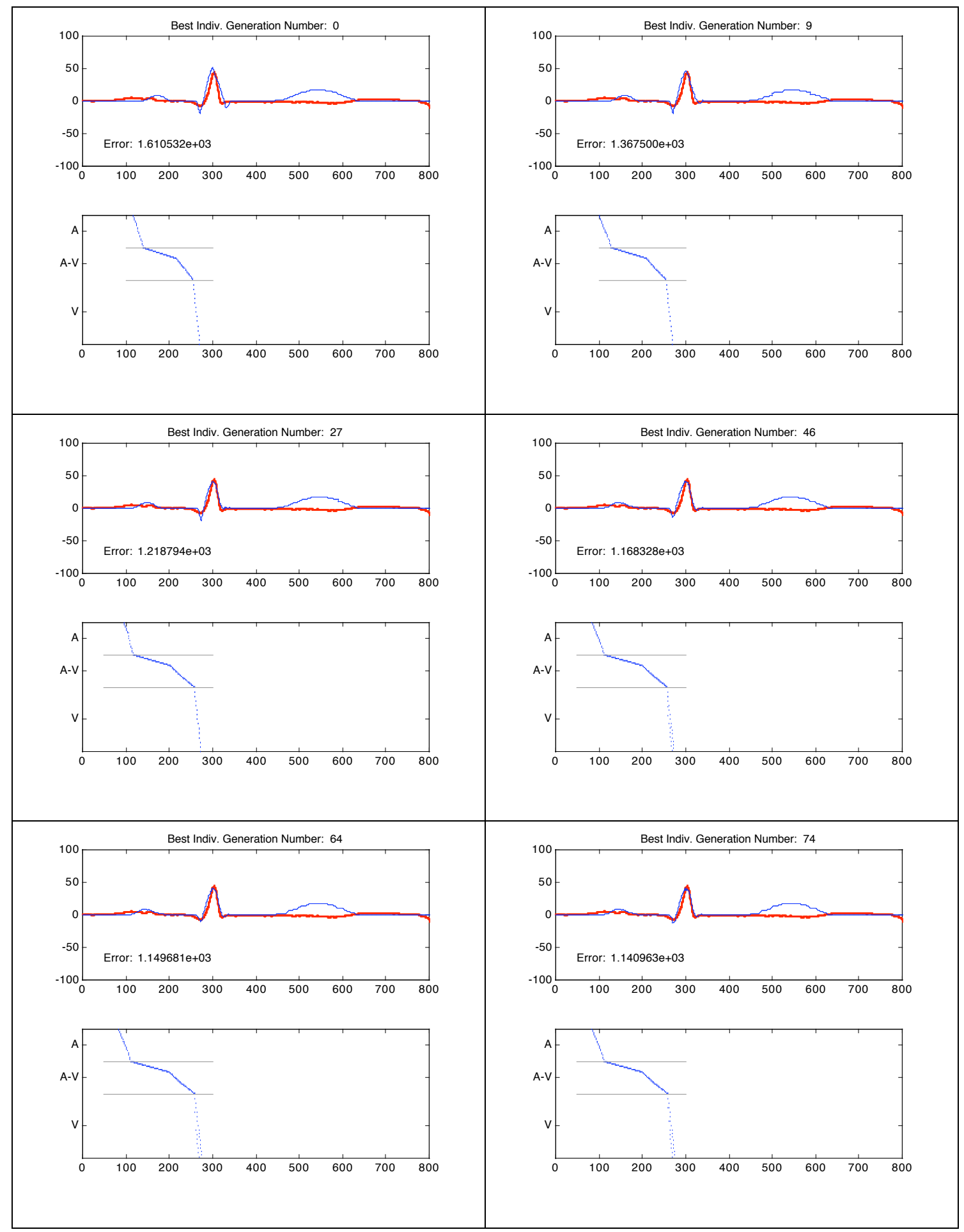

Figure 5. Model adaptation to a sinus beat in record 100 of the MIT-BIH database. Each panel shows the best individual of some populations of the EA, presenting the observed beat (thicker line) and the synthesized beat (finer line) along with its ladder diagram. 


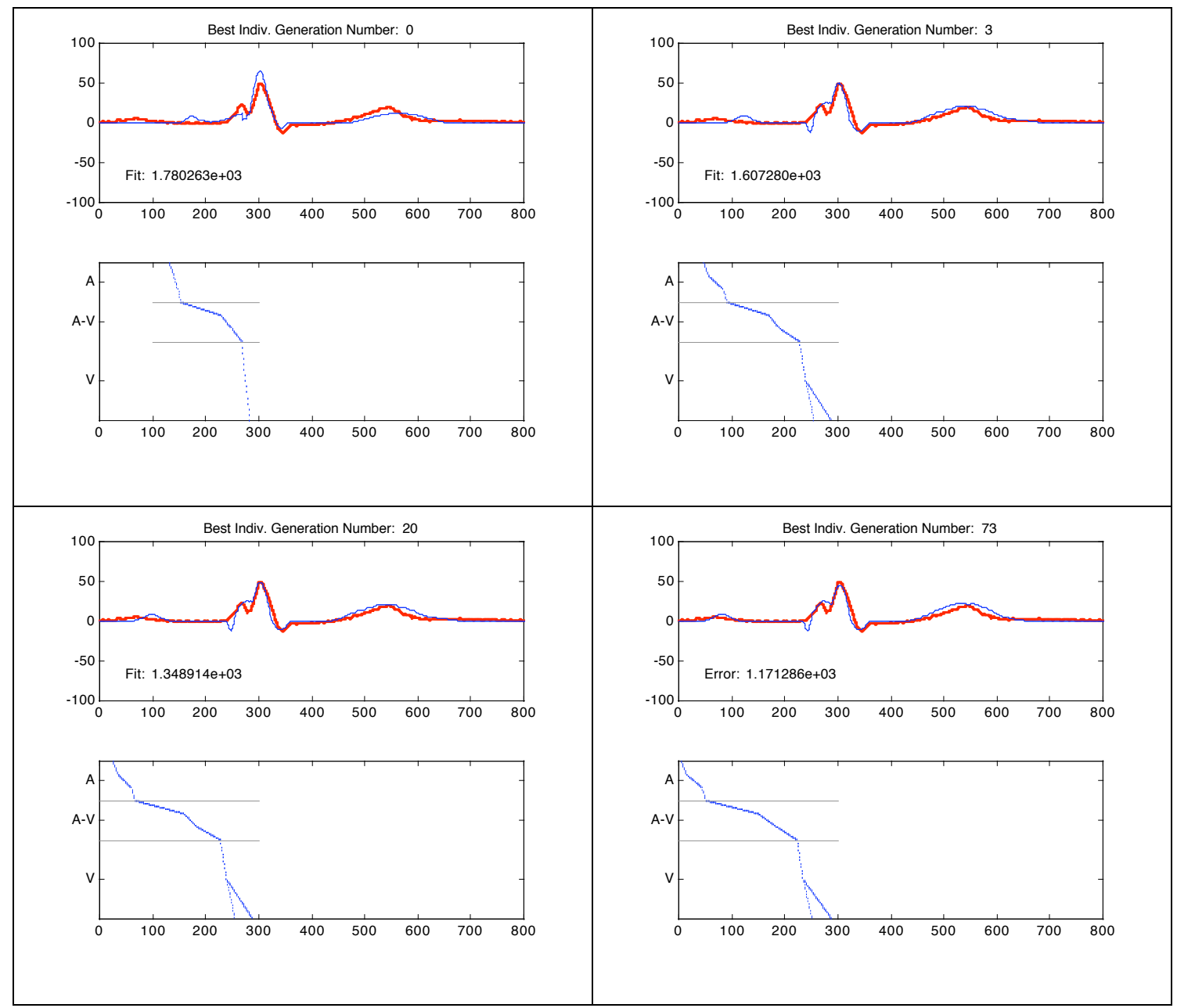

Figure 6. Adaptation of the model to a beat presenting a left bundle branch block obtained from record 111 of the MIT-BIH database. 


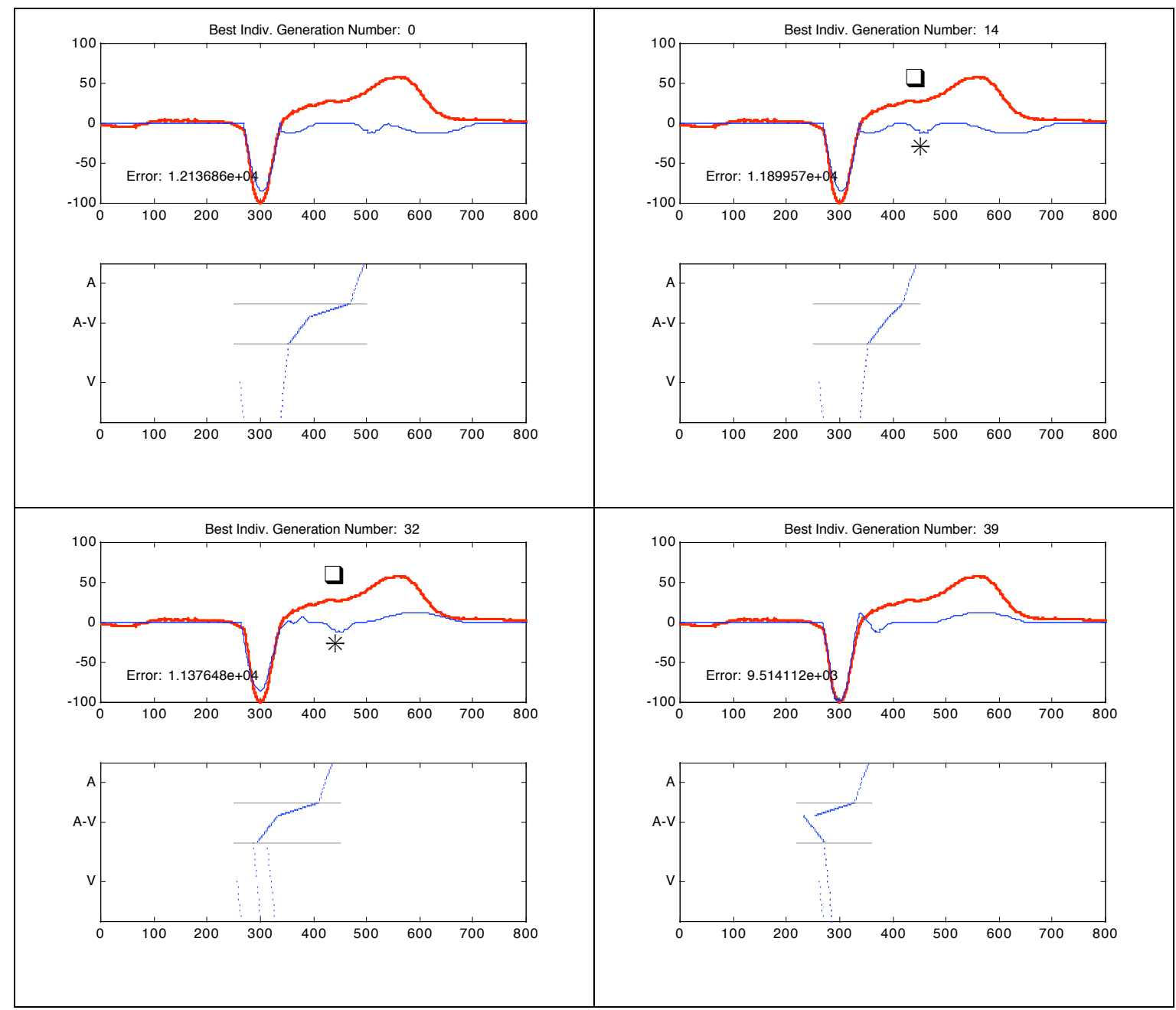

Figure 7. Application of the intra-beat adaptation algorithm to a premature ventricular contraction in record 100 of the MIT-BIH database. 


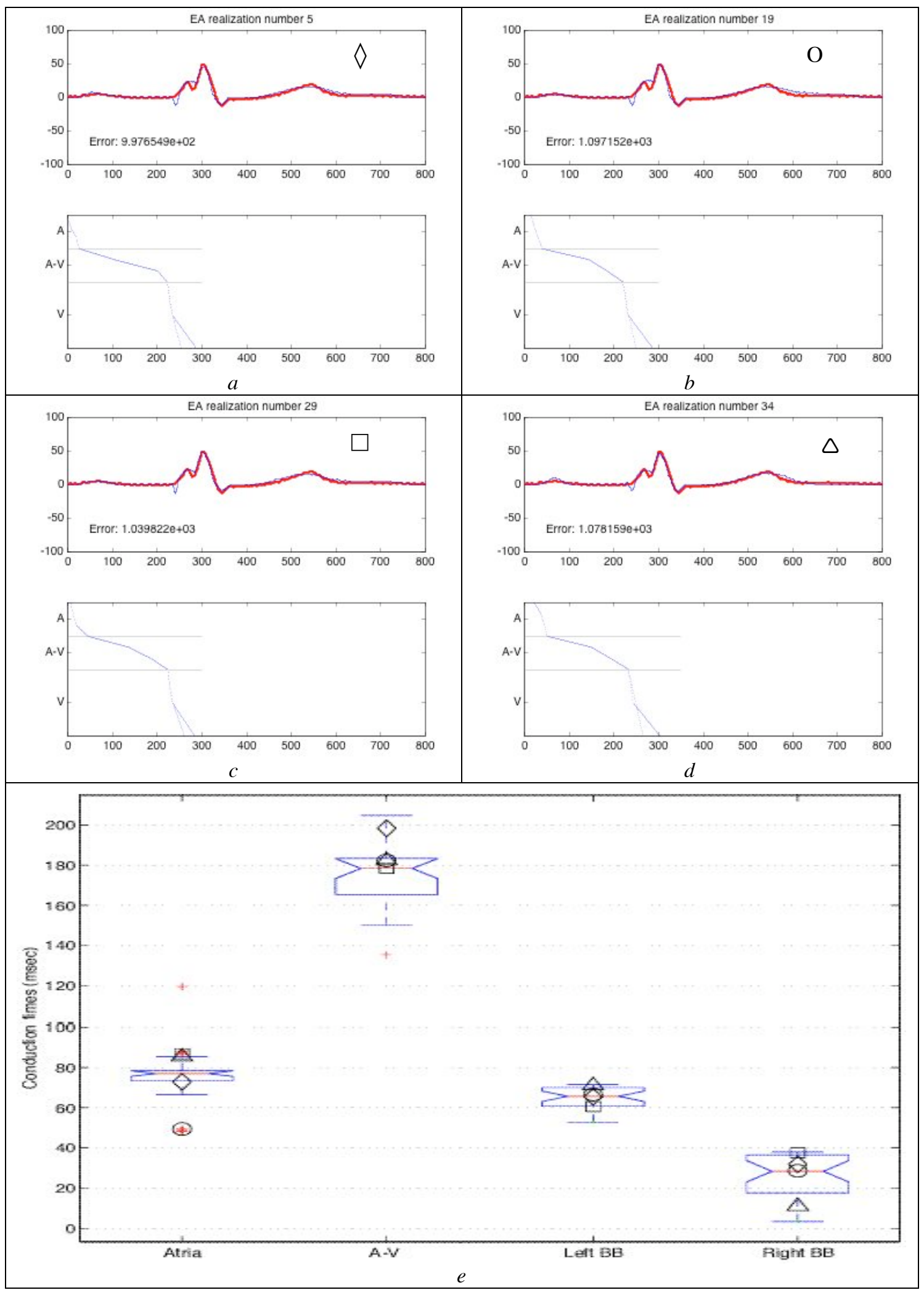

Figure 8. Panels $a$ to $d$ : Four solutions obtained from different realizations of the intra-beat adaptation algorithm for the same observed beat. Panel $e$ : Boxplot of conduction times through the atria, the atrio-ventricular junction and the left and right bundle branches, calculated from 35 different realizations of the intra-beat adaptation algorithm. The upper and lower parts of each box represent the last and the first quartile of the population, respectively; the line at the middle of the box represents the median value; the '+' symbol represents the outliers; the lines showing upper and lower limits symbolize the extent of the observed samples (without the outliers) and the notches show the $95 \%$ confidence interval of the mean value. Symbols $\nabla, O, \square$, and $\triangle$ show, respectively, the position of solutions $a, b, c$ and $d$ on the boxplot. 


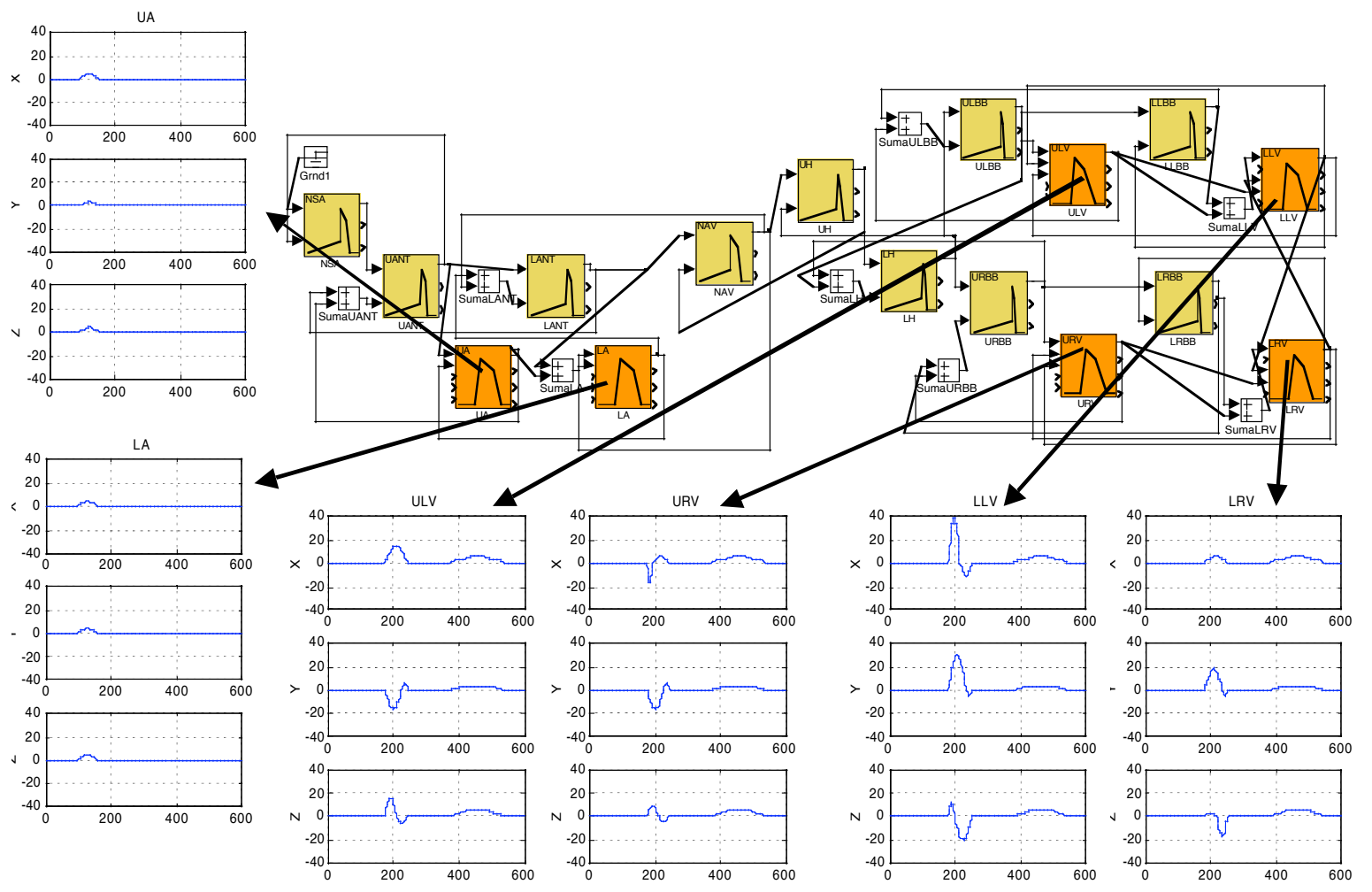

Figure 9. Simulink diagram of CARMEM's model (normal case without ectopic foci or accessory pathways) presenting the templates of each MA for the case of a normal antegrade conduction. 